\title{
Scaling of apparent stress from broadband radiated energy catalogue and seismic moment catalogue and its focal mechanism dependence
}

\author{
Z. L. Wu \\ Institute of Geophysics, China Seismological Bureau, 100081 Beijing, China \\ (Received November 20, 2000; Revised July 18, 2001; Accepted July 25, 2001)
}

\begin{abstract}
Comparison between the NEIC broadband radiated energy catalogue and the Harvard CMT catalogue provides information about the properties of apparent stress. Based on the numerical studies of Shaw (1998) we examined the ratio of broadband radiated energy and scalar seismic moment of shallow earthquakes worldwide from 1987 to 1998. It is observed that for different focal mechanisms, scaling of apparent stress or 'scaled energy' with seismic moment is different. For thrust and normal events, apparent stress decreases with seismic moment; while for strikeslip events, apparent stress increases with seismic moment. On average, for thrust events, the apparent stress of aftershocks is higher than that of main shocks; for strike-slip events, the apparent stress of aftershocks is lower than that of main shocks; while the situation of normal events is in between.
\end{abstract}

\section{Introduction}

Apparent stress (e.g., Wyss and Brune, 1968), as represented by

$$
\sigma_{\text {app }}=\eta \frac{E}{M_{0}}
$$

is one of the most important physical quantities describing a seismic source, providing information about the lower limit of the ambient stress level. In the above expression $\eta$ is the shear modulus of the source medium, $E$ the radiated energy, and $M_{0}$ the seismic moment. Before the $1980 \mathrm{~s}$, radiated energy $E$ and seismic moment $M_{0}$ can only be obtained through empirical relations between energy/moment and magnitudes, and thus could not be used directly in the analysis of apparent stress. Since recent years, development of broadband digital seismological observation has made it possible to measure radiated energy and seismic moment directly from the analysis of digital seismic waveforms, which makes it possible to have a better look at the properties of apparent stress and the physics behind. Using the NEIC broadband radiated energy estimates and the Harvard CMT results, Choy and Boatwright (1995) discussed the spatial distribution pattern of apparent stress, identifying different stress regimes related to global tectonics. By comparing radiated energy and seismic moment and calculating the energy budget, Kanamori et al. (1998) and Kanamori and Heaton (2000) proposed that there should be frictional meting in the rupture process of some of the large earthquakes. Newman and Okal (1998) used $E / M_{0}$ as a discriminant for tsunami earthquakes. Based on the temporal variation of seismic energy and apparent stress, Pulido and Irikura (2000) estimated the dynamic parameters which determine

Copy right (c) The Society of Geomagnetism and Earth, Planetary and Space Sciences (SGEPSS); The Seismological Society of Japan; The Volcanological Society of Japan; The Geodetic Society of Japan; The Japanese Society for Planetary Sciences. the properties of an earthquake rupture process. Scaling of apparent stress also provides useful clues about dynamic friction along an earthquake fault, as showed by the numerical modeling of Shaw (1998) whose basic idea we followed in this study.

It has to be recognized that, up to now, there are still significant uncertainties associated with the estimates of both radiated energy and seismic moment, therefore the result about apparent stress is still not very reliable. On the other hand, however, because of its importance in the dynamics of earthquakes, result about apparent stress, even if with limited reliability, is still worth discussing. In this paper we analyzed the global catalogue of the NEIC broadband radiated energy and the Harvard CMT to investigate the scaling properties of apparent stress. An observation being worth reporting is that earthquakes with different focal mechanisms exhibit different scaling of apparent stress, a phenomena which might be of importance in the physics of earthquakes.

\section{Data Used for the Analysis}

The concept of seismic moment tensor was introduced in the 1970s (Gilbert, 1970; Backus and Mulcahy, 1976, 1977; Backus, 1977a, b). Harvard University has started to publish centroid moment tensor (CMT) solutions for earthquakes with magnitude over 5.5 since the 1980s. Long-period body wave and/or mantle wave are used in the measurement of CMTs (Dziewonski et al., 1981). While still having some significant bias and/or uncertainty (e.g., Patton, 1998), CMT catalogue is widely used in many researches such as the frequency-moment relation (Frohlich and Davis, 1993; Sornette et al., 1996; Kagan, 1997), stress state and seismotectonics (e.g., Frohlich et al., 1997; Frohlich, 2000), global distribution of non-double-couple sources (Kawakatsu, 1991), clustering properties of earthquakes and 'seismic gap' (Kagan and Jackson, 1994, 1995), rate of foreshocks (Reasenberg, 1999), change of 'crustal potential energy' 


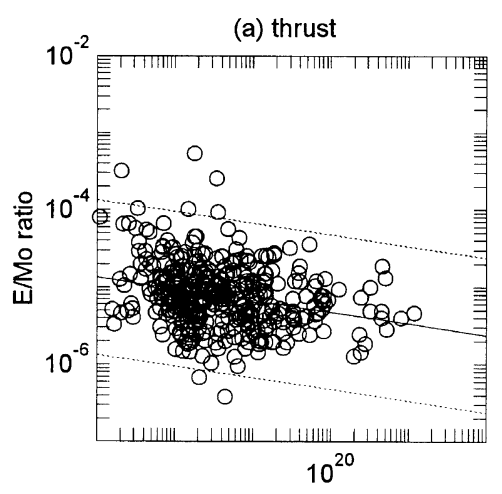

(b) strike-slip

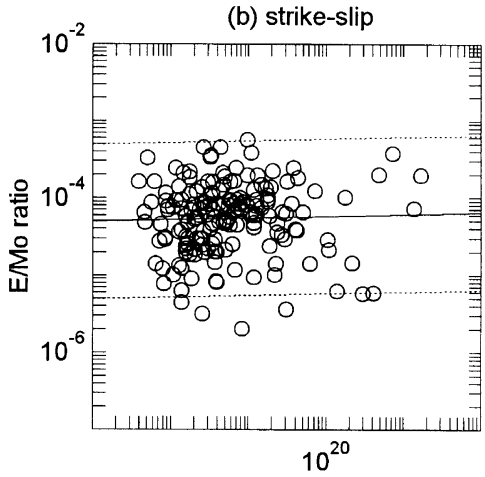

(c) normal

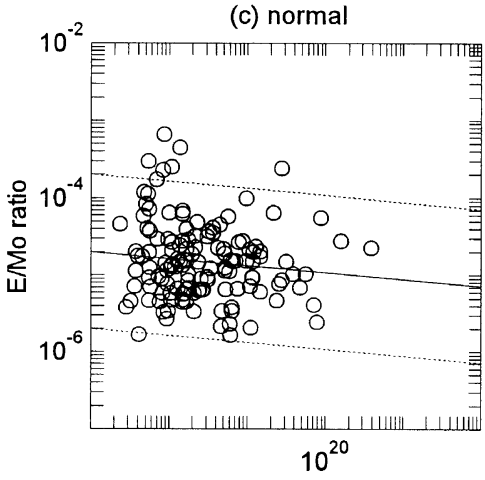

Seismic moment $\mathrm{Mo} / \mathrm{Nm}$

Fig. 1. Energy/moment ratio or 'scaled energy' versus seismic moment for shallow earthquakes worldwide from 1987 to 1998: thrust events (a); strike-slip events (b); and normal events (c).

(Tanimoto and Okamoto, 2000), 'earthquake doublets' (Kagan and Jackson, 1999), and temporal correlation of earthquake focal mechanisms (Kagan, 2000), among others. Harvard CMT catalogues are regularly published as research letters in Phys. Earth Planet. Inter, and are available through the web. In this paper, we use the CMT catalogue for global shallow earthquakes over M5.5 from January 1987 to December 1998.

The measurement of broadband radiated energy conducted by the National Earthquake Information Center (NEIC), U. S. Geological Survey was started in the 1980s (Boatwright and Choy, 1986; Choy and Boatwright, 1995). Teleseismic body waves are used in the measurement of broadband radiated energy. Broadband seismograms are retrieved from raw LP and SP data or from raw IP/BB data
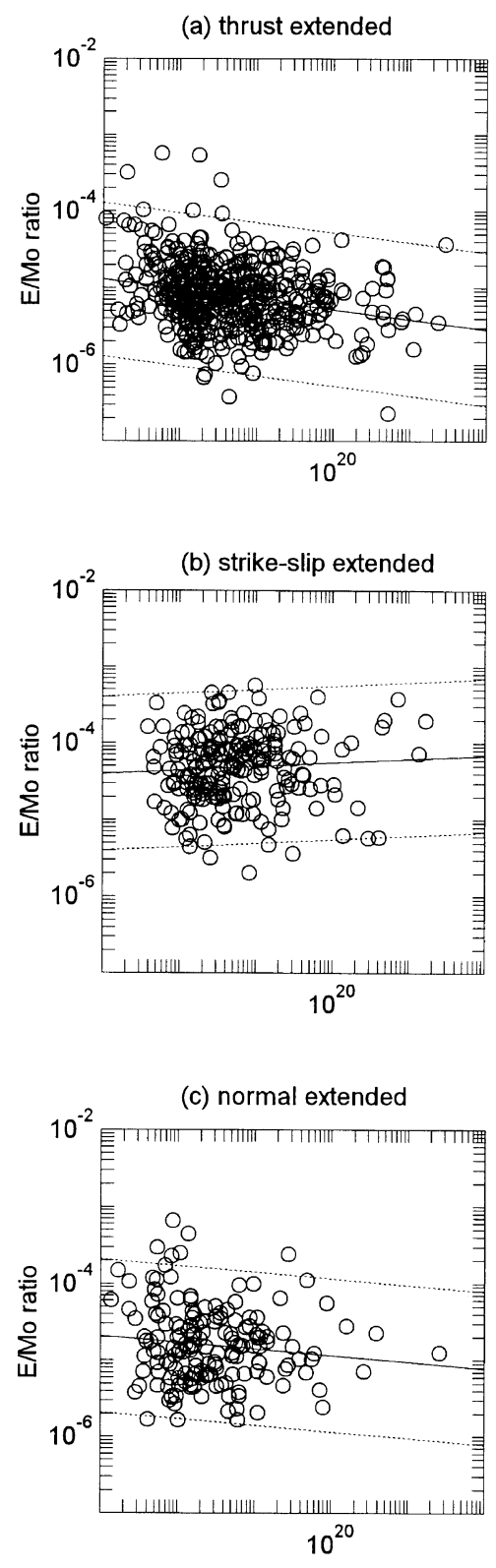

Seismic moment Mo/Nm

Fig. 2. Energy/moment ratio or 'scaled energy' versus seismic moment for shallow earthquakes worldwide from 1987 to 1998: 'extended' thrust events (a); 'extended' strike-slip events (b); and 'extended' normal events (c), see text for details.

using the algorithm proposed by Harvey and Choy (1982). Comparing to the Harvard CMT catalogue, the NEIC broadband radiated energy catalogue is still not widely used, although there are a few works related to apparent stress (Choy and Boatwright, 1995; Kanamori and Heaton, 2000). One of the reasons for this situation is that it is generally argued that broadband radiated energy is not necessarily a better measure of an earthquake than scalar seismic moment. But this argument is wrong: Broadband radiated energy, being derived from the velocity power spectra, is a measure of seismic potential for damage, while seismic moment, derived from the low-frequency asymptote of displacement spectra, is more physically related to the final displacement of an earthquake (Choy and Boatwright, 1995). Or in an- 


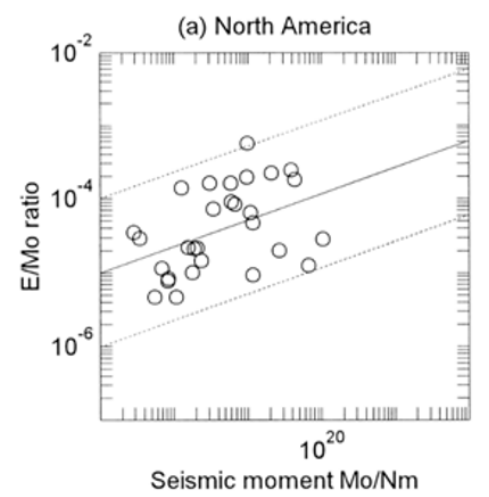

(c) North America

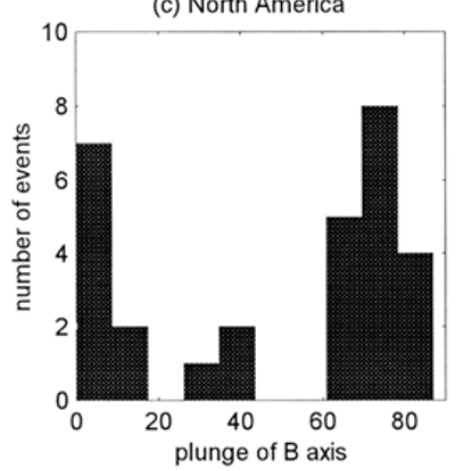

(b) Japan-Kuril

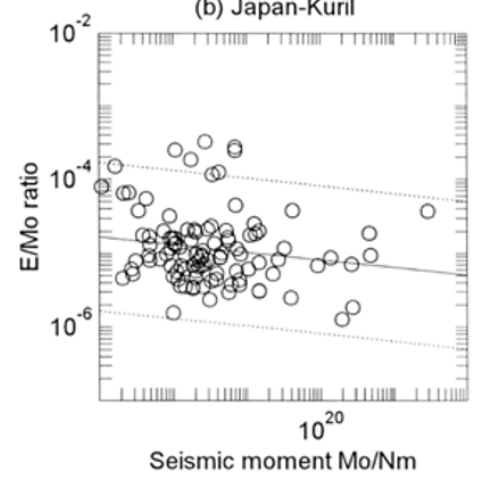

(d) Japan-Kuril

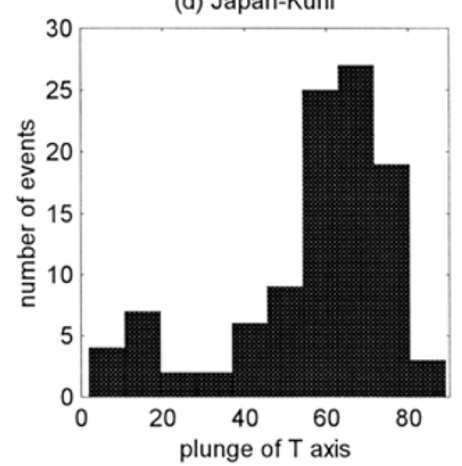

Fig. 3. Energy/moment ratio or 'scaled energy' versus seismic moment for shallow earthquakes from 1987 to 1998 : North America (a) and Japan-Kuril region (b). Histograms of the plunges of B or T axis as shown in (c) and (d) give the predominant focal mechanisms of the earthquakes under discussion.

other word, broadband radiated energy is not a better measure of an earthquake than seismic moment, it is rather an alternative measure other than seismic moment. In fact, some of the properties, such as the global frequency-size distribution of earthquakes, as represented by energy and moment, respectively, show completely different characteristics (Wu, 2000). NEIC broadband radiated energy catalogue was firstly published in the earthquake notes in Bull. Seism. Soc. $A m$. Since recent years the earthquake notes have changed to be published in Seism. Res. Lett. In this study, broadband radiated energy catalogue for global shallow earthquakes over M5.5 from January 1987 to December 1998 is used.

\section{Scaling of Apparent Stress versus Seismic Mo- ment}

Scaling of apparent stress versus seismic moment has significant importance in the microscopic and macroscopic physics of earthquakes (Shaw, 1998; Kanamori and Heaton, 2000). In such studies one of the more straightforward ways is to directly consider the ratio between energy and seismic moment, the so called 'scaled energy' (Kanamori and Heaton, 2000), which is equivalent to the assumption that the shear modulus of the earth medium is taken as the same for all the earthquakes. In the study we consider different focal mechanisms using the classification of Frohlich (1992). In such a classification, thrust, strike-slip, or normal mechanisms are defined as those with the plunges of $\mathrm{T}, \mathrm{B}$, or $\mathrm{P}$ axes larger than 60 degrees, respectively. In our analysis we also consider the 'extended thrust', 'extended strike-slip', and 'extended normal' events, which correspond to the events with the plunges of their T, B, or P axes larger than 45 de- grees, respectively.

Figure 1 shows the scaled energy versus seismic moment for shallow earthquakes worldwide with different focal mechanisms. Although the data is scattered, it can be seen that the scaled energy of thrust and normal events is on average lower than that of strike-slip events, a similar observation to that of Choy and Boatwright (1995) and PerezCampos and Beroza (2001). More importantly, it can be recognized that for thrust and normal events, the scaled energy decreases with seismic moment; while for strike-slip events, the scaled energy increases with seismic moment. Note that we are using a square coordinate system, so the tendency of increasing or decreasing, as shown in the figures, is evident. To characterize the changing tendency, we use a power law relation to fit the data. The fitting obtains that

$$
\begin{array}{lr}
E / M_{0} \propto M_{0}^{-0.15} & \text { for thrust events, } \\
E / M_{0} \propto M_{0}^{0.02} & \text { for strike-slip events, } \\
E / M_{0} \propto M_{0}^{-0.09} & \text { for normal events. }
\end{array}
$$

In the figure the solid line shows the fitting line, and the dotted lines show the range of uncertainty. It can be seen that although the uncertainty is up to 2 orders of magnitudes, the tendency of increasing or decreasing is clear. Figure 2 shows the result for the 'extended' mechanisms, and the changing tendency is similar.

To compare with previous results, we consider smaller regions. Figure 3 shows the scaled energy versus seismic moment for the region of $20^{\circ}-55^{\circ} \mathrm{N}, 105^{\circ}-140^{\circ} \mathrm{W}$, corresponding approximately to the north America, and the region of $30^{\circ}-60^{\circ} \mathrm{N}, 135^{\circ}-165^{\circ} \mathrm{E}$, corresponding approximately to the 
Japan-Kuril region, respectively. Histograms of the plunges of B or T axis, as represented by Figures 3(c) and 3(d), show that for the north America region, strike-slip mechanism is predominant, while for the Japan-Kuril region, thrust mechanism is predominant. The result shows that

$$
\begin{array}{lr}
E / M_{0} \propto M_{0}^{0.36} & \text { for north America, } \\
E / M_{0} \propto M_{0}^{-0.10} & \text { for Japan-Kuril region. }
\end{array}
$$

It is worth pointing out that, comparing the result of Kanamori and Heaton (2000) and this result, one may see that for the earthquakes in north America, the scaled energy has a tendency of increase with seismic moment. These two studies are in accordance with each other, although the result of Kanamori and Heaton (2000) is mainly for small to moderate events while this result is mainly for moderate to large events, and the result of Kanamori and Heaton (2000) is mainly for north America while this study deals with global seismicity.

\section{Apparent Stress of Aftershocks and Main Shocks}

To further confirm the above-mentioned observation, we consider subsets of the database in the last section. In this section we compare the energy/moment ratio or 'scaled energy' of aftershocks with that of main shocks. Comparing to the comparison in the last section, this comparison has an advantage because it is more reasonable to assume that the elastic modulus of earth medium in the source region of an aftershock is the same as that of the main shock. Therefore, to much extent, this comparison is equivalent to the comparison between the apparent stress of aftershocks and that of main shocks. Definition of aftershock is still an open question to discuss. However, for earthquakes with magnitude larger than 5.5, this question will not affect the result seriously. Based on the definition in other studies (e.g., Molchan and Dmitrieva, 1992), we take the earthquakes occurring after a bigger one within time $T$ and distance $D$ as an 'aftershock'. We take $T$ and $D$ to be linear functions of the magnitude $M$ of the main shock. When $M=5.0$, one has $T=0.5$ year and $D=50 \mathrm{~km}$; and when $M=7.0$, one has $T=1.5$ year and $D=150 \mathrm{~km}$. Figure 4 presents, for different focal mechanisms, respectively, the comparison between the apparent stress of the main shock $\sigma_{\text {app }}^{M}$ and that of its aftershock $\sigma_{\text {app }}^{A}$. In the figure the solid line shows $\sigma_{\text {app }}^{A}=\sigma_{\text {app }}^{M}$; data points above this line correspond to the events with apparent stress of aftershocks larger than that of main shocks, while data points below this line correspond to the events with apparent stress of aftershocks smaller than that of main shocks. Although the scatter of the data is large, even by visual inspection it can be seen that the difference between strike-slip and thrust earthquakes is evident. On average, for strike-slip earthquakes, the apparent stress of aftershocks is lower than that of main shocks; for thrust earthquakes, the apparent stress of aftershocks is higher than that of main shocks; while the case for normal events is in between. This is consistent with the conclusions drawn in the last section.

\section{Discussion and Conclusions}

Harvard CMT catalogue contains information of standard errors. The NEIC energy catalogue, however, provides in- formation of standard errors only before 1994; after 1995 such information does not appear in the catalogue. For these reason we do not give the information of uncertainties in Figures 1 to 5. Estimating on average, the order of magnitudes of the uncertainty for the 'scaled energy' $E / M_{0}$ is about $50 \%$. Another uncertain factor is the shear modulus of the earth medium, which is assumed to be the same for all the earthquakes under discussion. In the discussion of aftershocks, this uncertainty can be overcome to some extent, because it is reasonable to assume that the shear modulus of the earth medium in the source region of an aftershock is the same as that of the main shock. Both scalar seismic moment measurement and broadband radiated energy measurement may have systematic bias due to the corrections of the earth structure, seismic wave propagation/attenuation, and site re-
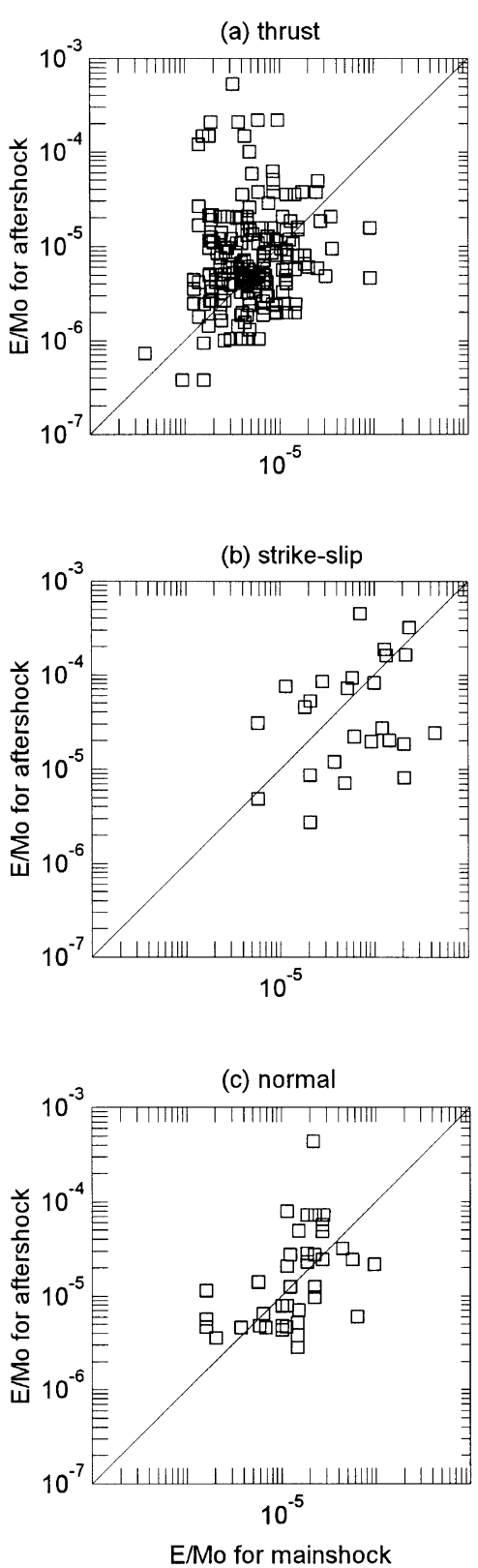

Fig. 4. Energy/moment ratio of aftershocks and main shocks: thrust events (a); strike-slip events (b); and normal events (c). 
sponse beneath each station. Taking all the above-mentioned factors into consideration, it can be concluded from the figures that the difference between strike-slip events and thrust events are evident, while the situation of normal events is marginal at present.

It is found that many of the earthquake phenomenology are dependent of focal mechanisms. For example, Reasenberg (1999) noticed that rate of foreshocks depends on the type of focal mechanisms. Frohlich and Davis (1993) observed that frequency-moment relation is different for different types of earthquakes. Tsuruoka et al. (1995) showed that tidal triggering effect of earthquakes is focal mechanism dependent. Choy and Boatwright (1995) and Perez-Campos and Beroza (2001) found that the average level of apparent
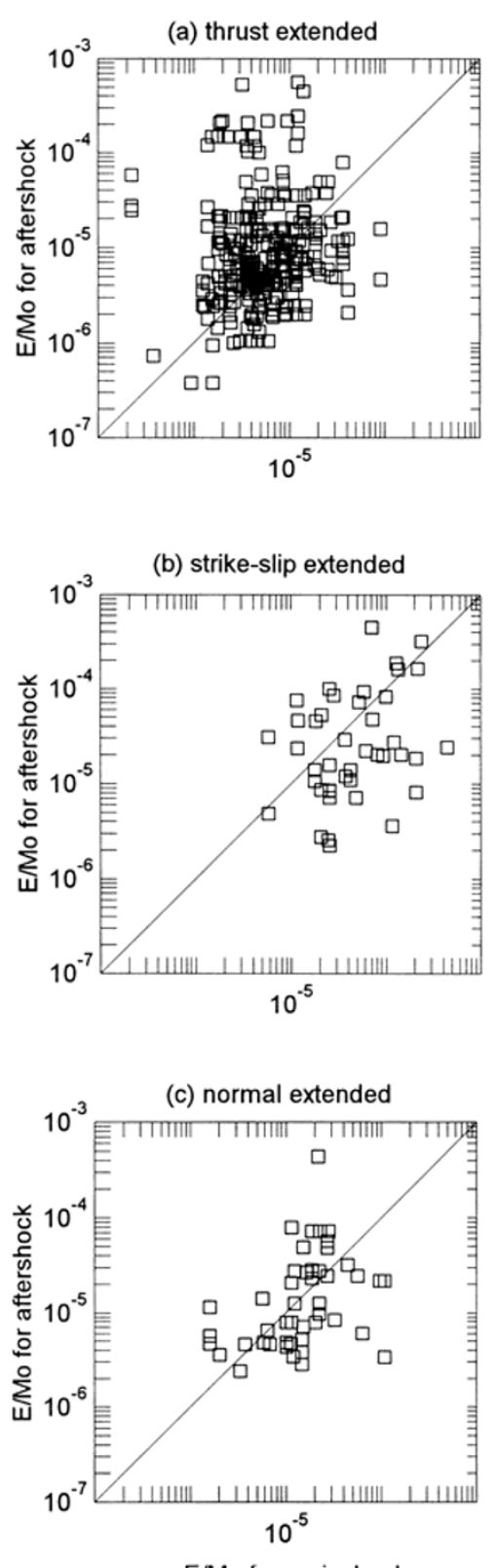

E/Mo for mainshock

Fig. 5. Energy/moment ratio of aftershocks and main shocks: 'extended' thrust events (a); 'extended' strike-slip events (b); and 'extended' normal events (c), see text for details. stress is different for earthquakes with different focal mechanism types, which can also be seen in Figures 1 and 2 in this paper. Once again in this study it is observed that the scaling of apparent stress versus seismic moment is different for earthquakes with different focal mechanisms: For thrust (and normal) events, apparent stress decreases with seismic moment, while for strike-slip events, apparent stress increases with seismic moment. On average, for thrust events, the apparent stress of aftershocks is higher than that of main shocks; while for strike-slip events, the apparent stress of aftershocks is lower than that of main shocks. There may be different explanations for this focal mechanism dependence, in the perspective of either seismotectonics or the physics of earthquakes. But it is important to notice that only using the 'modern earthquake catalogues' produced in the era of digital broadband seismology, such observations are feasible. Specially it is worth pointing out that the seismic moment catalogue and the broadband radiated energy catalogue are two parallel and independent 'modern earthquake catalogues,' being of the same importance. The comparison between these two catalogues may provide some interesting clues to the understanding of the nature of earthquakes.

Acknowledgments. Thanks are due to Prof. Y.-T. Chen for guidance in seismic source physics and Dr. G. Choy for providing the energy data. Comments and suggestions of the anonymous referees and Editor Prof. K. Yomogida are very helpful in improving the manuscript. This work is supported by Project G1998040705 and NSFC 49725410. The author is grateful to Lamont-Doherty Earth Observatory (LDEO), Columbia University, for support during his stay.

\section{References}

Backus, G. E., Interpreting the seismic glut moments of total degree two or less, Geophys. J. R. astron. Soc., 51, 1-25, 1977a.

Backus, G. E., Seismic sources with observable glut moments of spatial degree two, Geophys. J. R. astron. Soc., 51, 27-45, 1977 b.

Backus, G. and M. Mulcahy, Moment tensors and other phenomenological descriptions of seismic sources. I: continuous displacements, Geophys. J. R. astron. Soc., 46, 341-361, 1976.

Backus, G. and M. Mulcahy, Moment tensors and other phenomenological descriptions of seismic sources. II: discontinuous displacements, Geophys. J. R. astron. Soc., 47, 301-329, 1977.

Boatwright, J. and G. L. Choy, Teleseismic estimates of the energy radiated by shallow earthquakes, J. Geophys. Res., 91, 2095-2112, 1986.

Choy, G. L. and J. L. Boatwright, Global patterns of radiated seismic energy and apparent stress, J. Geophys. Res., 100, 18,205-18,288, 1995.

Dziewonski, A. M., T.-A. Chou, and J. H. Woodhouse, Determination of earthquake source parameters from waveform data for studies of global and regional seismicity, J. Geophys. Res., 86, 2825-2852, 1981.

Frohlich, C., Triangle diagrams: ternary graphs to display similarity and diversity of earthquake focal mechanisms, Phys. Earth Planet. Inter., $\mathbf{7 5}$, 193-198, 1992.

Frohlich, C., Display and quantitative assessment of distributions of earthquake focal mechanisms, Geophys. J. Int., 144, 300-308, 2000.

Frohlich, C. and S. D. Davis, Teleseismic $b$ values; or, much ado about 1.0, J. Geophys. Res., 98, 631-644, 1993.

Frohlich, C., M. F. Coffin, C. Massell, P. Mann, C. L. Schuur, S. D. Davis, T. Jones, and G. Karner, Constraints on Macquarie Ridge tectonics provided by Harvard focal mechanisms and teleseismic earthquake locations, J. Geophys. Res., 102, 5029-5041, 1997.

Gilbert, F., Exitation of the normal modes of the Earth by earthquake sources, Geophys. J. R. astron. Soc., 22, 223-226, 1970.

Harvey, D. and G. L. Choy, Broadband deconvolution of GDSN data, Geophys. J. R. astron. Soc., 69, 659-668, 1982.

Kagan, Y. Y., Seismic moment-frequency relation for shallow earthquakes: Regional comparison, J. Geophys. Res., 102, 2835-2852, 1997.

Kagan, Y. Y., Temporal correlations of earthquake focal mechanisms, Geophys. J. Int., 143, 881-897, 2000. 
Kagan, Y. Y. and D. D. Jackson, Long-term probabilistic forecasting of earthquakes, J. Geophys. Res., 99, 13,685-13,700, 1994.

Kagan, Y. Y. and D. D. Jackson, New seismic gap hypothesis: Five years after, J. Geophys. Res., 100, 3943-3959, 1995.

Kagan, Y. Y. and D. D. Jackson, Worldwide doublets of large shallow earthquakes, Bull. Seism. Soc. Am., 89, 1147-1155, 1999.

Kanamori, H. and T. H. Heaton, Microscopic and macroscopic physics of earthquakes, in GeoComplexity and the Physics of Earthquakes, edited by J. B. Rundle, D. L. Turcotte, and W. Klein, Washington, D. C., AGU, 147-163, 2000.

Kanamori, H., D. L. Anderson, and T. H. Heaton, Frictional melting during the rupture of the 1994 Bolivian earthquake, Science, 279, 839-842, 1998.

Kawakatsu, H., Enigma of earthquakes at ridge-transform-fault plate boundaries-distribution of non-double couple parameter of Harvard CMT solutions, Geophys. Res. Lett., 18, 1103-1106, 1991.

Molchan, G. M. and O. E. Dmitrieva, Aftershock identification: methods and new approaches, Geophys. J. Int., 109, 501-516, 1992.

Newman, A. V. and E. A. Okal, Teleseismic estimates of radiated seismic energy: The $E / M_{0}$ discriminant for tsunami earthquakes, J. Geophys. Res., 103, 26,885-26,898, 1998.

Patton, H. J., Bias in the centroid moment tensor for central Asian earthquakes: Evidence from regional surface wave data, J. Geophys. Res., 103, 26,963-26,974, 1998.

Perez-Campos, X. and G. C. Beroza, An apparent mechanism dependence of radiated seismic energy, J. Geophys. Res., 106, 11,127-11,136, 2001. Pulido, N. and K. Irikura, Estimation of dynamic rupture parameteres from the radiated seismic energy and apparent stress, Geophys. Res. Lett., 27, 3945-3948, 2000.

Reasenberg, P. A., Foreshock occurrence before large earthquakes, J. Geophys. Res., 104, 4755-4768, 1999.

Shaw, B., Far-field radiated energy scaling in elastodynamic earthquake fault models, Bull. Seism. Soc. Am., 88, 1457-1465, 1998.

Sornette, D., L. Knopoff, Y. Y. Kagan, and C. Vanneste, Rank-ordering statistics of extreme events: Application to the distribution of large earthquakes, J. Geophys. Res., 101, 13,883-13,893, 1996.

Tanimoto, T. and T. Okamoto, Change of crustal potential energy by earthquakes: an indicator for extensional and compressional tectonics, Geophys. Res. Lett., 27, 2313-2316, 2000.

Tsuruoka, H., M. Ohtake, and H. Sato, Statistical test of the tidal triggering of earthquakes: contribution of the ocean tide loading effect, Geophys. J. Int., 122, 183-194, 1995.

Wu, Z. L., Frequency-size distribution of global seismicity seen from broadband radiated energy, Geophys. J. Int., 142, 59-66, 2000.

Wyss, M. and J. N. Brune, Seismic moment, stress, and source dimensions for earthquakes in the California-Nevada region, J. Geophys. Res., 73 4681-4694, 1968.

Z. L.Wu (e-mail: wuzl@ns.cdsn.org.cn) 